\title{
The Effect of Strain on the Transport Properties of Superconducting Strand and Cable in a Conduit Conductor
}

\author{
Chao Zhou ${ }^{(0)}$, Marc Dhallé, Herman ten Kate, and Arend Nijhuis
}

\begin{abstract}
A proper understanding of the degradation of the transport properties of cable-in-conduit conductors (CICCs) due to a changing strain distribution or to crack formation in the filaments is essential to determine the operational limits of the conductors and to optimize their design. Based on the electrical and strain properties of the superconducting strand, the performance of short samples of the CICC can be analyzed, such as the ones tested in the SULTAN facility, or the full-size CICC used in real magnets. Mathematical fitting expressions are proposed to implement strand properties into the cable model. In combination with the strain maps generated by the mechanical model MULTIFIL, these expressions are introduced in the electromagnetic code JackPot to predict the current sharing temperature of the CICC of the international thermonuclear experimental reactor (ITER) Central Solenoid. A comparison is made with SULTAN short samples tests as well.
\end{abstract}

Index Terms-Cable-in-conduit conductor (CICC), strain, superconductivity.

\section{INTRODUCTION}

D EDICATED experiments and models are needed to quantitatively describe the behavior of composite conductors, in particular for large and complex cable-in-conduit conductors (CICCs), such as the ones used for the international thermonuclear experimental reactor (ITER) magnets [1]. Up to now, many experimental and theoretical studies have been devoted to analyze the effects of strain in $\mathrm{Nb}_{3} \mathrm{Sn}$ wires and multistrand conductors [2]-[6]. Several numerical cable models were developed for explaining a $\mathrm{Nb}_{3} \mathrm{Sn}$ CICC performance [7]-[11]. In [9] and [11], the effect of an axial compression was added to the periodically bending beam model for the analysis of the electromagnetic-mechanical behavior of CICCs for high magnetic field applications. However, without proper inputs to the models regarding the mechanical and electromagnetic

Manuscript received April 16, 2018; revised July 16, 2018; accepted July 16, 2018. Date of publication July 24, 2018; date of current version August 2, 2018. This paper was recommended by Associate Editor L. Chiesa. (Corresponding author: Chao Zhou.)

C. Zhou, M. Dhallé, and A. Nijhuis are with the Department of Energy, Materials and Systems, Faculty of Science and Technology, University of Twente, Enschede 7500AE, The Netherlands (e-mail: c.zhou@utwente.nl).

H. ten Kate is with the Department of Energy, Materials and Systems, Faculty of Science and Technology, University of Twente, Enschede 7500AE, The Netherlands, and also with CERN, Geneva 1211, Switzerland.

Color versions of one or more of the figures in this paper are available online at http://ieeexplore.ieee.org.

Digital Object Identifier 10.1109/TASC.2018.2858758 properties of superconducting strands and cables, the outcome remains merely qualitative [1].

Furthermore, to quantitatively understand the complex phenomena in a cables comprising strain-sensitive material like $\mathrm{Nb}_{3} \mathrm{Sn}$, the electromagnetic and mechanical models of the CICCs need to be coupled. The Twente three-dimensional (3-D) strand model provides an understanding of an strand performance under different loads [12] together with the in-depth intrastrand resistance investigation [13]. The MULTIFIL model [14], a finite element mechanical code developed at the Ecole Centrale de Paris, Gif-sur-Yvette, France, can assess the strain distribution in multistage superconducting cables. That allows computing the strain state of strands imposed both by the cabling process and by the Lorentz forces experienced during the operation [14]. The JackPot code, a numerical tool for the electromagnetic simulation of CICCs, is based on the following individual strand trajectories and uses measured data to derive all network parameters [15]. The only remaining free variable in JackPot is the local strain state of the $\mathrm{Nb}_{3} \mathrm{Sn}$ strands, which can be calculated with MULTIFIL. The flexibility of JackPot allows us to implement different $I_{\mathrm{c}}(T, B, \varepsilon)$ critical current scaling laws. The ITER scaling law [16] takes into account an axial strain, but a scaling law also including bending strain is still missing and required to complete the coupled electromagnetic and mechanical cable models (JackPot-MULTIFIL). A number of mathematical expressions are proposed to link strand characteristics with a cable model that accounts for both axial and bending strain, as well as for a filament fracture. The preliminary results of such a novel electromagnetic-mechanical model are presented and analyzed.

\section{EFFeCtive Mathematical EXPRESSIONS From AN STRAND TO A CABLE MODEL}

Implementing the local critical current $I_{\mathrm{c}}$ and $n$-value, as well as the detailed strain state of individual filaments in a strand in the JackPot model, which already is simulating many hundreds of strands, would make the computation time prohibitively long. Instead, a drastic simplification is proposed by which a multifilamentary wire subjected to strain variation is described as a monofilament strand that experiences a combination of neutralaxis axial strain and peak bending strain, as illustrated in Figs. 1 and 2. The neutral-axis axial strain and peak bending strain of the monofilament strand are figured out by obtaining the same 

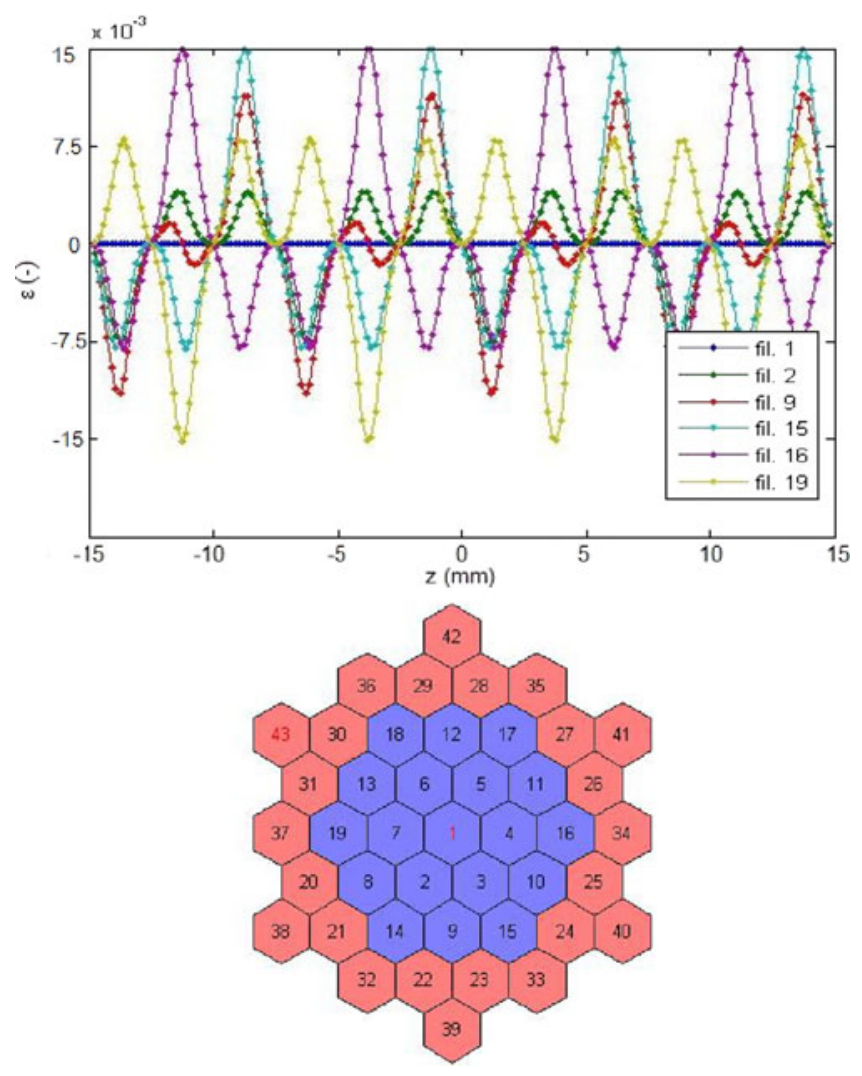

Fig. 1. Strain versus axial position showing the longitudinal strain distribution in various filaments (with twist pitch of $15 \mathrm{~mm}$, picked from different rings of the filamentary zone) under periodic bending with a peak bending strain of $1.5 \%$. The filament numbers refer to the bottom picture of the cross section of an internal-tin $\mathrm{Nb}_{3} \mathrm{Sn}$ strand (the blue cells are superconducting filaments, and the red ones represent the copper sheath).

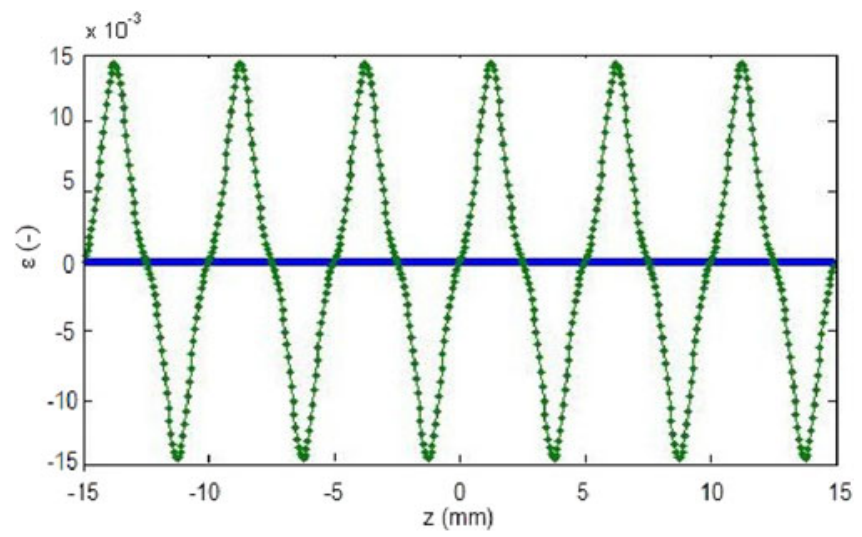

Fig. 2. Proposed longitudinal strain distribution in the simplified filamentary region under periodic bending with a peak bending strain of $1.5 \%$. The blue line represents the neutral-axis axial strain $(0 \%)$.

$I_{\mathrm{c}}$ performance from the ITER scaling law [16] as that of the multifilament strand, with the 3-D strand model [12].

In order to construct a mathematical expression for the combined effect of neutral-axis axial strain and bending strain, the bending strain is assumed to be uniform over a short distance $(\Delta x \rightarrow 0)$ along the strand, while the strain distribution in the transverse direction varies linearly from tensile to compressive. With such a uniform bending strain assumption, the

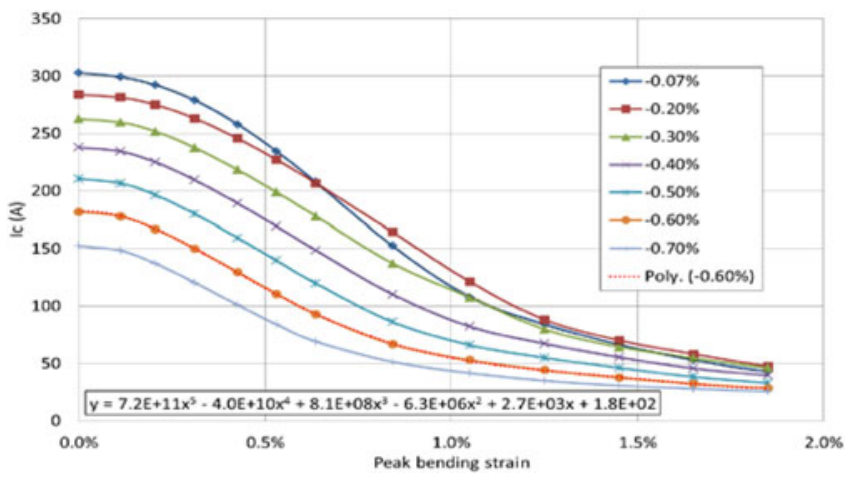

Fig. 3. Critical current of a "virtual" internal-tin strand as a function of uniform peak bending strain at $4.2 \mathrm{~K}$ and $12 \mathrm{~T}$. The different symbols represent various axial strain values $\varepsilon_{\mathrm{a} 0}$ applied to the neutral bending axis. The polynomial function at $\varepsilon_{a}=-0.6 \%$ is indicated as an example.

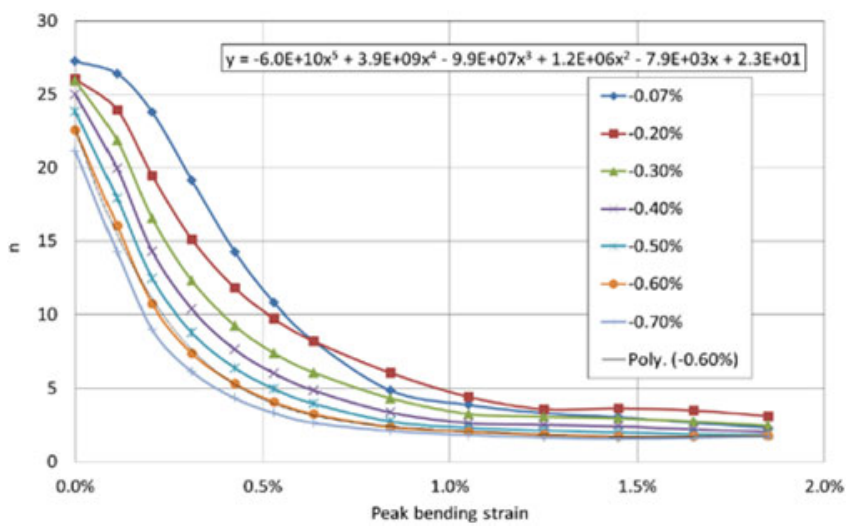

Fig. 4. $n$-value of a "virtual" internal-tin strand as a function of uniform peak bending strain at $4.2 \mathrm{~K}$ and $12 \mathrm{~T}$. The different symbols represent various axial strain values $\varepsilon_{\mathrm{a} 0}$ applied to the neutral bending axis. The polynomial function at $\varepsilon_{a 0}=-0.6 \%$ is indicated as an example.

$I_{\mathrm{c}}$ and $n$-value versus uniform peak bending strain at various levels of neutral-axis axial strain are shown in Figs. 3 and 4, calculated with the 3-D strand model [12] for an internal-tin strand at $4.2 \mathrm{~K}$ and $12 \mathrm{~T}$. Both $I_{\mathrm{c}}$ and $n$-value as a function of the peak bending strain can be represented by polynomial fitting functions.

As an example, the polynomial function at $-0.6 \%$ neutralaxis axial strain $\left(\varepsilon_{a 0}\right)$ is written in each plot. With this approach, the complex $I_{\mathrm{C}}$ distribution in the filaments under periodic bending (see Fig. 5) can be simplified to an $I_{\mathrm{c}}$ distribution in an equivalent mono-filamentary wire subjected to periodic bending (see Fig. 6), which can then easily be implemented into a cable model such as JackPot as a modified scaling law that includes both axial and bending strain. It should be noted that current redistribution from one strand section to the next is neglected in this approach. This is allowed since the length required for current sharing between strands is several orders of magnitude higher than for intrastrand redistribution [17], [18].

In order to evaluate the effect of this simplification, a comparison is made between experimental test arrangement for strain influence on strands (TARSIS) bending data [1], [19] and the predictions of the strand model with the extracted functions 


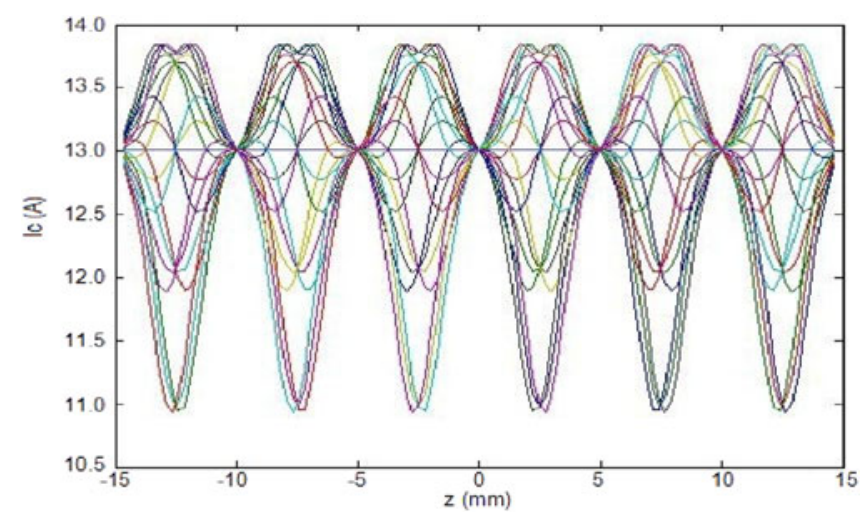

Fig. 5. Local filament critical current $I_{\mathrm{c}}$ distribution along all filaments under periodic bending with a peak bending strain of $0.1 \%$ in an internal-tin wire.

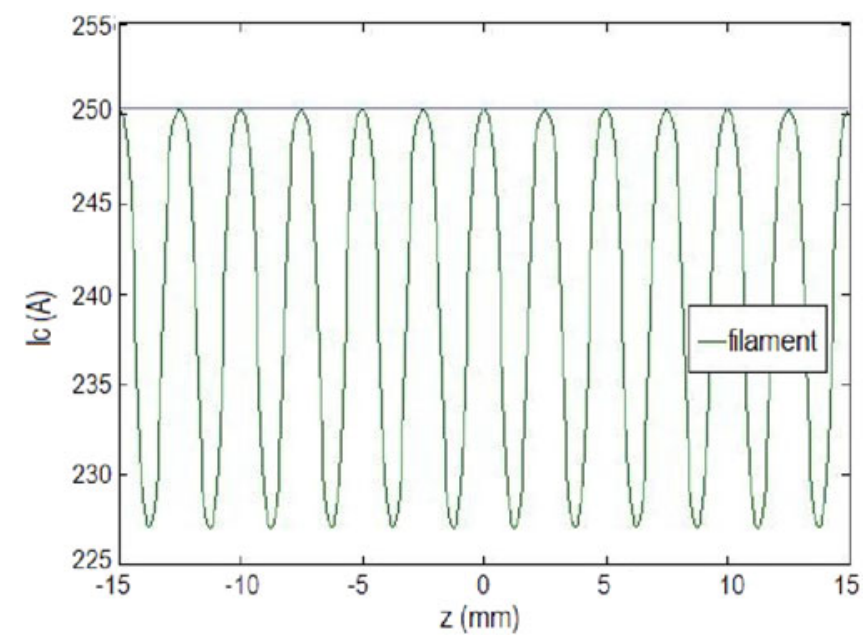

Fig. 6. Critical current versus axial position showing the longitudinal $I_{\mathrm{c}}$ distribution of the "virtual" internal-tin strand with simplified filamentary region under periodic bending (peak bending strain of $0.1 \%$ and neutral-axis axial strain of $0 \%$ ).

for $I_{\mathrm{c}}$ and $n$-values versus uniform peak bending strain implemented.

As shown in Fig. 7, $I_{\mathrm{c}}$ deviates around $20 \%$ in the high peak bending strain region. Since the $n$-value is not well predicted by the 3-D strand model [12], an $n$-value is applied following the ITER scaling law using the calculated $I_{\mathrm{c}}$ with the mathematical functions, showing a much better agreement (see Fig. 7). Finally, the $n$-value is also empirically determined fitting the predicted and measured results (called modified $n$ in Fig. 7, blue line). These three methods to determine the $n$-value will be compared also in the coupled electromagnetic and mechanical cable model to gain a better understanding of the detailed influence of the $n$-value on the behavior under bending loads.

Also, the influence of magnetic field and temperature is investigated with the 3-D strand model [12]. Normalized $I_{\mathrm{c}}$ and $n$-values follow the same curves with the same bending strain pattern for different magnetic field and temperature. The normalized curves $I_{\mathrm{c}}\left(\varepsilon_{p b}\right) / I_{\mathrm{c}}(0)=f_{I}\left(\varepsilon_{p b}\right)$ and $n\left(\varepsilon_{p b}\right) / n(0)=$ $f n\left(\varepsilon_{p b}\right)$ as a function of uniform peak bending strain under neutral-axis axial strain $\varepsilon_{a 0}=-0.6 \%$ for an internal-tin strand

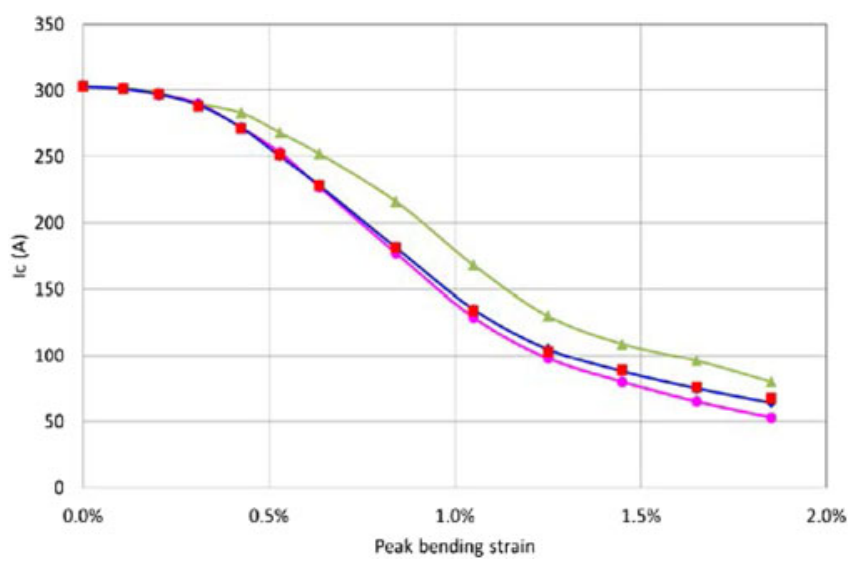

Fig. 7. Critical current as a function of a periodic peak bending strain measured on an internal-tin strand in a TARSIS experiment (red squares). The data are compared to a simulation that uses the fitting polynomials for $I_{\mathrm{c}}$ and $n$ with the uniform bending assumption (green line); $n$-values from the ITER scaling law using the uniform bending $I_{\mathrm{c}}$ (purple line); or the modified functions with uniform bending (blue line).

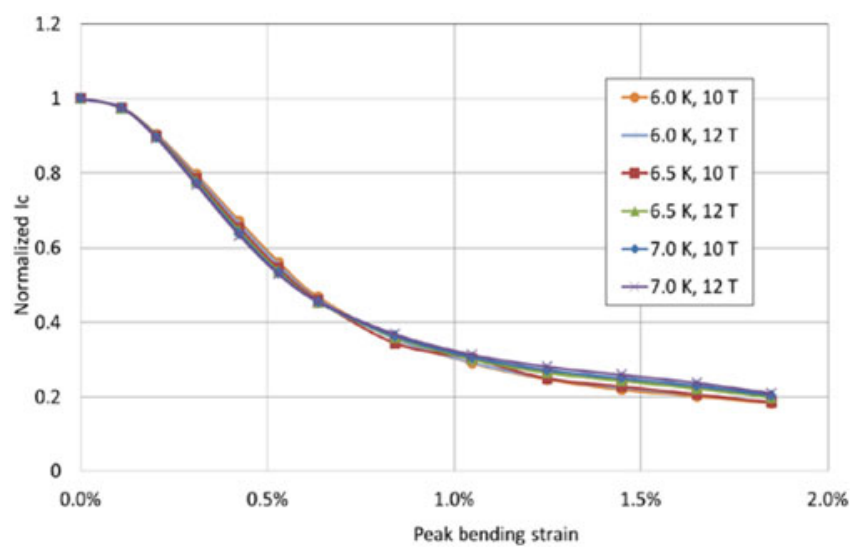

Fig. 8. Normalized critical current versus uniform peak bending strain at various temperatures and magnetic fields, simulated with the 3-D strand model for an internal-tin strand at $\varepsilon_{a 0}=-0.6 \%$.

are shown at various temperatures and magnetic fields in Figs. 8 and 9. The curves are virtually independent of magnetic field and temperature. This is coherent with the ITER scaling law for axial strain, where the shape of the $I_{\mathrm{c}}(\varepsilon)$ curves of $\mathrm{Nb}_{3} \mathrm{Sn}$ conductors is only marginally affected by the magnetic field and temperature [16].

This observation allows us to separate the mathematical expression $I_{\mathrm{c}}(B, T, \varepsilon)$ for the coupled electromagnetic and mechanical cable model into an axial-strain dependent part $I_{c 0}\left(B, T, \varepsilon_{a 0}\right)$ multiplied by the bending-strain dependent function $f_{I}\left(\varepsilon_{p b}\right)$ determined at a given value of $\varepsilon_{a 0}$ as

$$
I_{c}(B, T, \varepsilon)=I_{c 0}\left(B, T, \varepsilon_{a 0}\right) * f_{I}\left(\varepsilon_{p b}\right) \mid \varepsilon_{a 0}
$$

where $\varepsilon$ represents the combined axial and bending strain. $I_{c 0}\left(B, T, \varepsilon_{a 0}\right)$ is obtained from an ITER scaling law, while $f_{I}\left(\varepsilon_{p b}\right) \mid \varepsilon_{a 0}$ is the polynomial function that can be fitted to the normalized $I_{\mathrm{c}}$ versus uniform peak bending strain $\left(\varepsilon_{p b}\right)$ curve for the corresponding neutral-axis axial strain $\left(\varepsilon_{a 0}\right)$ (see Fig. 10). 


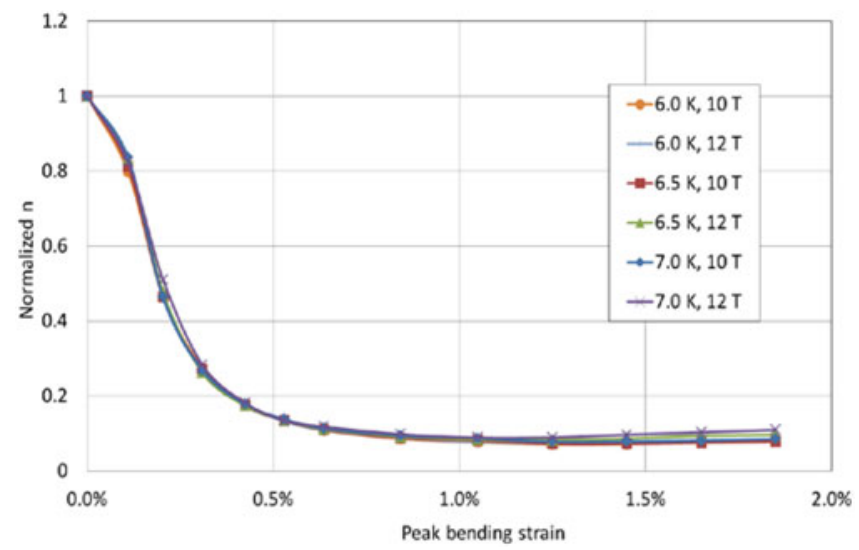

Fig. 9. Normalized $n$-value versus uniform peak bending strain at various temperatures and magnetic fields, simulated with the 3-D strand model for an internal-tin strand at $\varepsilon_{a 0}=-0.6 \%$.

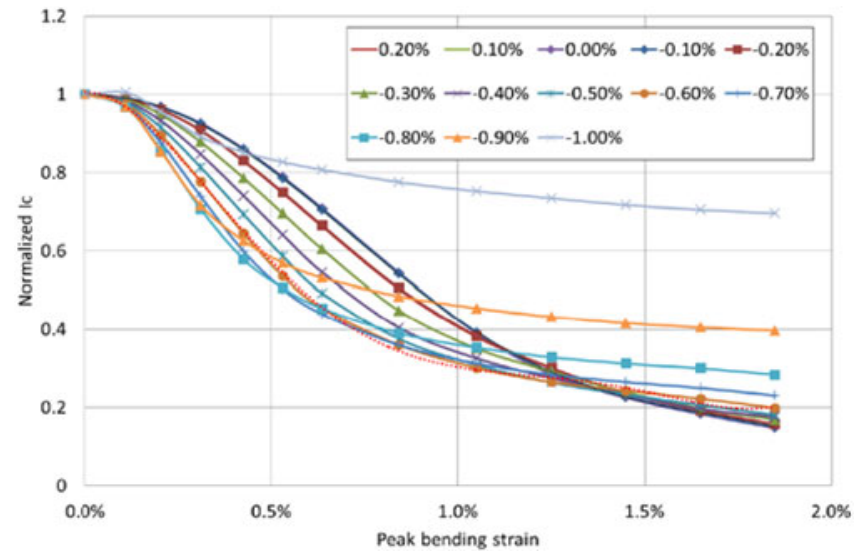

Fig. 10. Normalized critical current as a function of uniform peak bending strain, simulated for an internal-tin strand at various levels of neutral-axis axial strain $\varepsilon_{\text {a } 0}$ (from $0.2 \%$ down to $-1.0 \%$ with each step of $0.1 \%$ ). The polynomial fitting expression for each $\varepsilon_{a 0}$ is the sought-for $f_{I}\left(\varepsilon_{p b}\right) \mid \varepsilon_{a 0}$.

Note that the ITER scaling law predictions do not always fit the University of Twente Pacman data [1], [20] perfectly, especially in the highly tensile strain region where filament crack occurs (see Fig. 11, blue diamond symbols). As the strands in CICC after cooling down are mainly subjected under compression, the crack occurs in high tensile strain and, thus, does not significantly affect the CICC $T_{\mathrm{cs}}$.

In combination with the strain maps of MULTIFIL and these "linking" polynomials, the JackPot model is able to predict a cable performance quantitatively in terms of the electric field versus temperature curves and also current sharing temperature $T_{\mathrm{cs}}$, when exposed to a combined thermal and Lorentz force load. Examples are shown in Fig. 12. The main characteristics of the so-called "Baseline," "Long Twist Pitch," and "Short Twist Pitch" (STP) cables are described in [14]. The differences are mainly in the detailed cable pattern and in the twist pitch sequence used for each cabling stage, especially the twist pitch of first stage CICC. In Fig. 12, "scaling (1)" represents the ITER scaling law [16] based on the Pacman data with only axial strain present, while "scaling (2)" uses the polynomials described above to incorporate the effect of

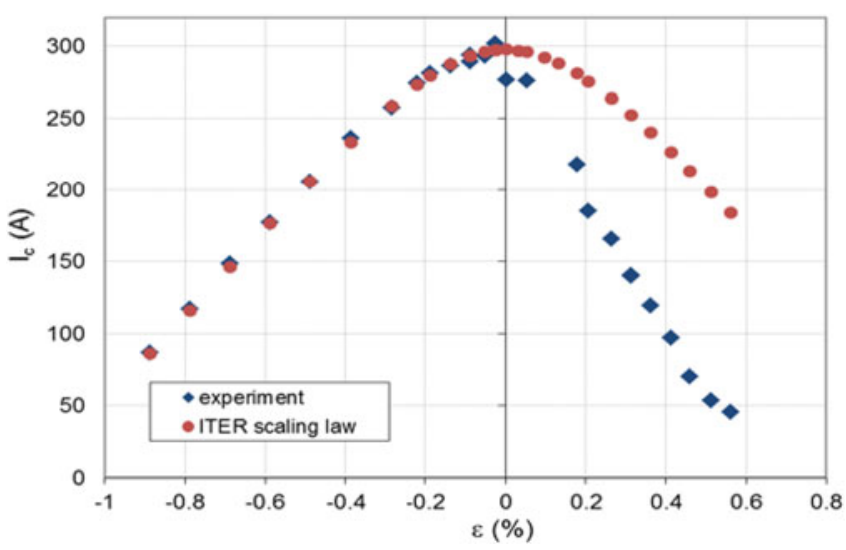

Fig. 11. Axial strain dependence of the critical current predicted by the ITER scaling law and observed in a Pacman test on an internal-tin strand at $4.2 \mathrm{~K}$ and $12 \mathrm{~T}$. The deviation is caused by an irreversible $I_{\mathrm{c}}$ degradation due to the filament cracks.

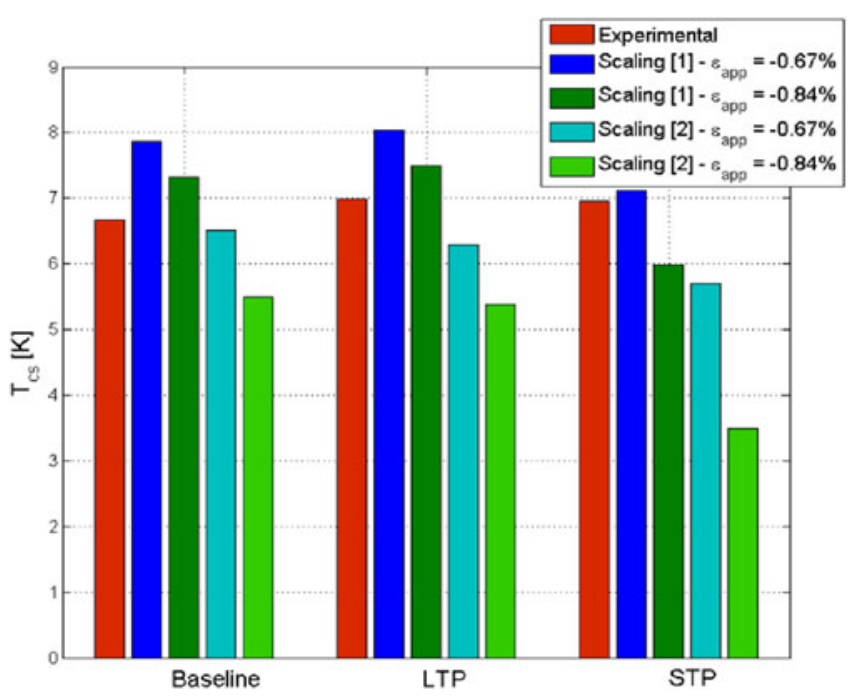

Fig. 12. Measured and simulated current sharing temperature $T_{\mathrm{cs}}$ for three different types of cables with a length of $150 \mathrm{~mm}$ (the CICC length in highest field zone, i.e., with a highest electromagnetic force in SULTAN test.), $-0.67 \%$ and $-0.84 \%$ applied axial strain. The calculations are made with the coupled cable model JackPot + MULTIFIL, using two different strand scaling laws.

bending strain [Equation (2)]. $n$-values are calculated from the modified functions with uniform bending (blue line in Fig. 7). The applied effective axial strain (e.g., $-0.67 \%$ and $-0.84 \%$ ) is obtained from the MULTIFIL model, which is the average axial strain of all the strand's combined $\varepsilon_{a 0}$ and $\varepsilon_{p b}$ in the CICC.

Both scaling laws can easily be implemented in JackPot in combination with MULTIFIL. After taking into account also the effects of bending strain and filament fracture, instead of only considering the effective axial stain [10], the current sharing temperature $T_{\mathrm{cs}}$ is reduced by about $1 \mathrm{~K}$. As shown in Fig. 12, for the Baseline cable, the $T_{\mathrm{cs}}$ calculated with scaling Equation (2) is closer to the measured data than with scaling Equation (1). However, the opposite is true for the STP cable. It is, thus, too early to conclude that the proposed polynomial expressions are more effective for predicting cable performance 
when compared to the standard scaling expression. At present, the combined electromagnetic and mechanical modeling does not reproduce all experimental results well. The mathematical expressions that link the strand and cable behavior cannot yet be convincingly validated. An improved cable strain map and better-characterized $n$-values under bending load may, however, lead to a better quantitative prediction.

\section{CONCLUSION}

Several mathematical expressions, polynomial fitting functions including the dependence of the critical current and $n$-value on temperature, magnetic field, axial strain, peak bending strain, and crack density, are proposed to link the appropriate strand properties to the combined electro-magnetic and mechanical cable models that are required to provide a quantitative prediction of conductor performance. Further investigation and work on a mechanical strain map of the CICC and a combined electromechanical model are needed for a better $T_{\mathrm{cS}}$ quantitative analysis, e.g., a detailed strain map of all strands in CICC by steps (in terms of cabling, compaction, thermal loads of heattreatment and cooling down, and electromagnetic loads), as well as better-characterized $n$-value both from an $I_{\mathrm{c}}$ measurement and an strand model prediction.

\section{REFERENCE}

[1] A. Nijhuis et al., "The effect of axial and transverse loading on the transport properties of ITER $\mathrm{Nb}_{3}$ Sn strands," Supercond. Sci. Technol., vol. 26, 2013, Art. no. 084004.

[2] N. Mitchell, "Operating strain effects in $\mathrm{Nb}_{3} \mathrm{Sn}$ cable-in-conduit conductors," Supercond. Sci. Technol., vol. 18, p. S396, 2005.

[3] D. Bessette, "Sensitivity of $\mathrm{Nb}_{3}$ Sn ITER conductor design to selected parameter," IEEE Trans. Appl. Supercond., vol. 13, no. 2, pp. 1433-1436, Jun. 2003.

[4] A. Nijhuis et al., "Optimization of interstrand coupling loss and transverse load degradation in ITER $\mathrm{Nb}_{3}$ Sn CICCs," IEEE Trans. Appl. Supercond., vol. 23, no. 3, Jun. 2013, Art. no. 4201206.

[5] A. Devred, C. Jong, and N. Mitchell, "Strain redistribution effects on current-sharing measurements on straight samples of large $\mathrm{Nb}_{3} \mathrm{Sn}$ cablein-conduit conductors," Supercond. Sci. Technol., vol. 25, 2012, Art. no. 054009 .
[6] P. Bruzzone et al., "Upgrade of operating range for SULTAN test facility," IEEE Trans. Appl. Supercond., vol. 12, no. 1, pp. 520-523, Mar. 2002.

[7] N. Mitchell, "Analysis of the effect of $\mathrm{Nb}_{3} \mathrm{Sn}$ strand bending on CICC superconductor performance," Cryogenics, vol. 42, pp. 311-325, 2002.

[8] A. Nijhuis and Y. Ilyin, "Spatial periodic contact stress and critical current of a $\mathrm{Nb}_{3}$ Sn strand measured in TARSIS," Supercond. Sci. Technol., vol. 19, pp. 945-962, 2006.

[9] Y. Zhai and M. D. Bird, "Florida electro-mechanical cable model of $\mathrm{Nb}_{3} \mathrm{Sn}$ CICCs for high-field magnet design," Supercond. Sci. Technol., vol. 21, 2008, Art. no. 115010.

[10] M. Breschi et al., "Modeling of the electro-mechanical behavior of ITER $\mathrm{Nb}_{3} \mathrm{Sn}$ cable in conduit conductors," Supercond. Sci. Technol., vol. 25, 2012, Art. no. 054005

[11] Y. Zhai, "Electro-mechanical modeling of $\mathrm{Nb}_{3} \mathrm{Sn}$ CICC performance degradation due to strand bending and inter-filament current transfer," Cryogenics, vol. 50, pp. 149-157, 2010.

[12] Y. Miyoshi et al., "Modelling of current distribution in $\mathrm{Nb}_{3} \mathrm{Sn}$ multifilamentary strands subjected to bending," Supercond. Sci. Technol., vol. 25, 2012, Art. no. 054003.

[13] C. Zhou et al., "Inter-filament resistance, effective transverse resistivity and coupling loss in superconducting multi-filamentary $\mathrm{NbTi}$ and $\mathrm{Nb}_{3} \mathrm{Sn}$ strands," Supercond. Sci. Technol. vol. 25, 2012, Art. no. 015013.

[14] H. Bajas, "Numerical simulation of the mechanical behavior of the ITER cable-in-conduit conductors," Ph.D. dissertation, Ecole Centrale de Paris, Gif-sur-Yvette, France, 2012.

[15] E. P. A. van Lanen and A. Nijhuis, "Numerical analysis of the DC performance of ITER TF samples with different cabling pattern based on resistance measurements on terminations," Supercond. Sci. Technol., vol. 24, 2011, Art. no. 085010 .

[16] A. Godeke et al., "A general scaling relation for the critical current density in $\mathrm{Nb}_{3}$ Sn wire," Supercond. Sci. Technol. vol. 19, p. R100, 2006.

[17] C. Zhou, M. Dhallé, H. H. J. ten Kate, and A. Nijhuis, "Current transfer length in multi-filamentary superconducting $\mathrm{NbTi}$ and $\mathrm{Nb}_{3} \mathrm{Sn}$ strands Experiments and models," Supercond. Sci. Technol., vol. 27, 2014, Art. no. 095014.

[18] A. Nijhuis et al., "The effect of inter-bundle resistive barriers on coupling loss, current distribution and DC performance in ITER conductors," IEEE Trans. Appl. Supercond., vol. 16, no. 2, pp. 868-871, Jun. 2006.

[19] A. Nijhuis et al., "Critical current and strand stiffness of three types of $\mathrm{Nb}_{3} \mathrm{Sn}$ strand subjected to spatial periodic bending," Supercond. Sci. Technol., vol. 19, pp. 1136-1145, 2006.

[20] A. Godeke et al., "A device to investigate the axial strain dependence of the critical current density in superconductors," Rev. Sci. Instrum., vol. 75 , no. 12, pp. 5112-5118, 2004.

Authors' biographies not available at the time of publication. 